portional to the number of sheets of paper applied, that is, to the thickness of the sheet of sediments. If the cover of paper is omitted and the other conditions are unaltered, the experiment produces the faults of the adjacent Basin and Ranges field. This variation corresponds exactly to the natural model.

Consequently, the tectonic deformations of the crust and the main features of its surface history can be more easily and better understood if we do not connect the visible structures directly with the supposed underground forces (subcrustal currents, convection as an effect of thermodynamic alterations, etc.). It is simpler to introduce an anisotropic and heterogeneous aggregate of pre-formed units which act as supports for the superficial material and as points of attack, as levers, and as instruments of transportation for the underground causal forces.

\section{PRESERVATION OF THE KAURI IN NEW ZEALAND}

$\mathrm{I}_{z}^{\mathrm{N}}$ a series of short newspaper articles in New Zealand, Prof. V. J. Chapman (professor of botany at Auckland University College) gives the conclusions he has reached on a problem of much interest to botanists and tree-lovers the world over, namely, the preservation of that remarkable tree, the kauri (Agathis australis Salisb.).

The only surviving extensive block of natural forest carrying big kauri is Waipoua Forest, covering some 30,000 acres and in the care of the State Forest Service. Here the kauri, including trees of outstanding size, occurs scattered throughout, mainly in groups. It is claimed that this tree may exceed even the Sequoias in timber content, owing largely to the remarkable way in which the trunk holds to the cylindrical form instead of tapering considerably towards the crown. Younger trees and saplings are to be found, but only in limited numbers. Experimental sowings or plantings have not always been successful, and though the N.Z. Forest Service is now more or less prepared to guarantee regeneration of this species, its critics do not yet appear convinced -a matter which it should be easy to settle on the spot.

Two main questions have arisen: What steps should be taken to ensure the protestion of an adequate number of the existing big trees and the perpetuation of the species; and if for this purpose areas are excluded from green fellings, should dead and dying trees be utilized before they decay ? It is alleged that the Forest Service has not hitherto publicly guaranteed complete protection from interference for any part of the forest, not even for the roadside strip, and that when felling for timber, usually takes all trees down to $30 \mathrm{in}$. in diameter and all dead and unhealthy trees fit for milling.

In Prof. Chapman's party, when he made the inspection of the forest on which his articles are based, the botanical and amenity interests were strongly represented, but unfortunately there does not appear to have been an experienced forester with them. To get the disinterested forestry point of view, he referred the matter as just another instance of a general problem to the sylviculturist of the Indian Forest Research Institute, and to the present reviewer, who has for many years campaigned among his colleagues and others for the preservation of outstanding trees and adequate samples of the forest types of which they form part: neither knew that Prof. Chapman intended publication. Meanwhile, the New Zealand Institute of Foresters has published a well-reasoned account of its view $(J . N . Z$. Inst. Forest., 5, No. 3, 173-175 ; 1946).

There seems to be agreement that a portion or portions of the forest should be excluded from fellings with suitable safeguards against change of policy, as has already been done by the N.Z. Forest Service in a working plan already six years old. All living trees on a fairly wide strip on either side of the State highway should be, and indeed are, to be kept untouched in this way, for in New Zealand, as in Great Britain, the truth is that appreciative as a large section of the general public may be of woodland amenities and big trees, it rarely goes far from the road. A certain number of exceptional trees should obviously also be specially protected, and access provided : this also has already been decided on.

Scientific interests, including those of forestry itself, call for the preservation of a further compact block of forest (not a narrow strip), which would be excluded from ordinary management. One or two areas of about 250-500 acres each might be suggested, and there is a good case in favour of leaving in them even dead trees standing or fallen, as it is very likely indeed that some part of the fauna is dependent on the dead and decaying wood for its existence and survival. We understand one such area has already been set aside for the purpose.

Over the rest of the forest, normal management for timber production can be permitted. It is remarkable how fully the amenities of a forest area can be maintained with such management where it is the settled policy so to maintain the:s : the New Forest in England provides an example, thanks to the care of the deputy surveyor, for it is almost incredible that more than two dozen sawmills were working there throughout most of the war-years with so little consequent loss to the amenities or even change in appearance. Prof. Chapman urges that in the managed portion, kauri should be retained as the principal species, just as in Britain it has been decided in certain forests that broad-leaved trees should not be replaced by conifers : we believe this to be desirable and that the State Forest Service is willing to agree. But just how that will be $\operatorname{don} \theta$ is not yet known, for on the information before us the kauri is a baffling tree, and a good deal more systematic research work will have to be done before it can be regenerated to order. Recorded information makes it clear that rather special conditions are required for the successful germination of the seed and establishment of the resultant plant; it is, however, not yet adequately known what those conditions are, though, as with many other trees, a primary requirement appears to be exposure of the mineral soil from its excessive covering of humus. The grouped occurrence, though by no means a unique feature in forest vegetation, has repeatedly been described, and studies are needed on soil and vegetational differences inside the groups and outside : the kauri, of course, itself influences the soil in which it grows and on which it showers its dead leaves and twigs. There is also a question of fungal association with the roots, which may incidentally play some part in the difficulties experienced in the nursery with this species. The importance of greater concentration on this research is underlined by the recommendation that until it is well under way only dead timber should be removed. 
We feel sure that agreement will soon be reached in these matters, as there are no serious difficulties in meeting the requirements of both sides in the controversy. The forester of to-day has been trained to realize the amenity and biological aspects of the handling of forests equally with the timber-getting and economic aspects. The New Zealand Forest Service is fully competent to apply the agreed policy to this important forest and is the obvious authority to do so: its research staff is expanding, and though the problems presented by this exceptionally interesting tree are perhaps complex, they should be quite capable of solution in a few years. Meanwhile, it is as well that keen interest should be taken in the preservation for future generations of one of the most remarkable trees of the world.

H. G. Champion

\section{ELECTRICAL PROPERTIES OF SOIL AND WATER AT CENTIMETRE WAVE-LENGTHS}

$\mathrm{T}$ HE electrical properties of the materials forming the earth's surface have always been of interest to the radio physicist and communication engineer, since the propagation of long and medium waves is markedly dependent upon the absorbing effect of the ground. At shorter wave-lengths, for which the radiation can be transmitted more freely through the lower or upper atmosphere, the reflexion coefficient of the earth's surface plays an important part in the propagation of the waves. More recently, the varying reflexion coefficient due to the different electrical properties of the different portions of the earth's surface has been used with conspicuous success in a radar method of navigation.

At moderate radio frequencies, the appropriate properties of soil and water can be determined by placing samples of the material in question in a condenser of suitable size and shape; and the results of many measurements made in this way at frequencies up to $100 \mathrm{Mc} / \mathrm{s}$. have been described by R. L. Smith-Rose ${ }^{1}$. A laboratory method of measuring the electrical properties of salt-water solutions has also been used by $R$. Cooper ${ }^{2}$ at frequencies up to more than 4,000 Mc./s., corresponding to wave. lengths down to $7 \mathrm{~cm}$. Alternatively, the reflexion coefficient of the surface of the ground can be measured directly, for example, by the standingwave method developed by J. S. MePetrie ${ }^{3}$, who with J. A. Saxton ${ }^{4}$ has used this technique at various frequencies up to about $600 \mathrm{Mc}$./s. (wave-length $50 \mathrm{~cm}$.). At the still higher frequencies of $3,300 \mathrm{Mc} . / \mathrm{s}$., corresponding to a wave-length of $9 \mathrm{~cm}$., L. H. Ford and $R$. Oliver ${ }^{5}$ have investigated the direct reflexion of radio waves from a wide range of surfaces including bare ground, both level and in ridges, ground covered with vegetation, fresh water and salt water approximating in composition to sea water. More recently, this free radiation technique has been extended by J. A. Saxton and J. A. Lane $\theta^{6}$ to the experimental determination of the dielectric properties of water for wave-lengths of 1.24 and $1.58 \mathrm{~cm}$.

With the growth and development of radar techniques in recent years, the above-mentioned British work has been supplemented by the work of other investigators, notably in the United States, some of whom have paid particular attention to the measurement of the reflexion coefficient of sea water at the small grazing angles met with in radar practice.
A report, which has recently been submitted to Nature, describes measurements of the dielectric properties of soils and water at a wave-length of $3 \cdot 2 \mathrm{~cm}$. (frequency $9,400 \mathrm{Mc} . / \mathrm{s}$.) carried out at the University of Texas as part of the programme of the United States Office of Naval Research. The report is by Prof. A. W. Straiton and C. W. Tolbert, and is of interest in so far as particular attention was devoted to the measurement of the properties of the dry, sandy soil found in Arizona where the Navy Electronics Laboratory had a site for special radio experiments. The measurements were made by placing samples of the material in a wave-guide or other suitable circuit arrangement, and measuring the phase-difference and attenuation caused by transmission through different thicknesses of the material. From such observations the dielectric constant and conductivity of the dry Arizona soil were found to be 3.2 and $10^{\circ}$ E.S.U. respectively. (A conductivity of $10^{10}$ E.S.U. corresponds to $1 \cdot 1$ Mhos/metre.) Similar measurements made on dry sandy loam taken from near the University Laboratory in Texas gave values for the dielectric constant of $2 \cdot 8$ and $0.7 \times 10^{8}$ E.S.U. for the conductivity. The authors of the report point out that the Arizona soil shows a conductivity of about three times as high as that obtained by Ford and Oliver ${ }^{5}$ for very dry sandy loam in Great Britain, while the value for the Texas soil is much smaller. It is suggested that the considerable iron content of the Arizona soil may account for the higher conductivity in this case. This is scarcely consistent, however, with the results of further measurements made by the authors on samples of the Arizona soil sifted through screens of various sizes of mesh. The samples of smallest size of particle contained no free iron, but showed the highest conductivity. Furthermore, measurements made on the sample of medium-size particles varied from $6 \times 10^{8}$ E.S.U. when loosely packed into the container to more than $10 \times 10^{8}$ E.S.U. when very tightly packed. As was the case for measurements made by earlier investigators, the conductivity of the soils from both Arizona and Texas was found to increase several times when the samples were moistened with fresh water.

In a somewhat similar way, measurements were made under the same conditions on distilled water, and on a sample of sea water from the Gulf of Mexico. For the fresh water, at a temperature of $23^{\circ} \mathrm{C}$., the dielectric constant was found to be 67 and the conductivity about $10^{11}$ E.S.U. (12 Mhos/metre); while for the sea water, which had a salt content of $2 \cdot 2$ per cent, the values obtained were 65 and $15 \times$ $10^{10}$ E.S.U. (16 Mhos/metre) respectively for a temperature of $28^{\circ} \mathrm{C}$. These values are in reasonably good agreement with the values deduced by Saxton? from a theoretical study of the anomalous dispersion of water combined with the results of measurements made at shorter wave-lengths than those used by Straiton and Tolbert. This recent work thus appears to form a useful extension of our knowledge of the electrical properties of soil and water at the frequencies corresponding to very short radio wave-lengths.

${ }^{1}$ Smith-Rose, R. I., J. Inst. Elect. Eng., 75, 221 (1934); Proc. Phys. Soc., 47, 923 (i935).

${ }^{2}$ Cooper, R., J. Inst. Elect. Eng., 93, III, 69 (1946).

$\checkmark$ McPetrie, J. S., Proc. Phys. Soc., 46, 637 (1934).

- McPetrie, J. S., and Saxton, J. A., J. Inst. Elect. Eng., 90, III, 33 (1943); 92, III, 256 (1945)

5 Ford, L. H., and Oliver, R., Proc. Phys. Soc., 58, 265 (1946).

- Saxton, J. A., and Lane, J. A., Conf. Rept. Phys. Soc. and Roy. Met. Soc., 278 (1946).

${ }^{7}$ Saxton, J. A., Conf. Rept. Phys. Soc. and Roy. Met. Soc., 292 (1946). 